\title{
Mycobacterium tuberculosis Culture Supernatant Induces Cancer Cell Apoptosis and Cell Cycle Arrest
}

\author{
Chao $\mathrm{Ma}^{1,2}$, Jiangbing Zhou ${ }^{1}$, Xiaolu Yin ${ }^{1}$, Chunfa $\mathrm{Jie}^{3}$, Dongming Xing ${ }^{2}$, Lijun $\mathrm{Du}^{2}$ and \\ Ying Zhang ${ }^{*}, 1$
}

\author{
${ }^{1}$ Department of Molecular Microbiology and Immunology, Bloomberg School of Public Health, Johns Hopkins \\ University, Baltimore, MD 21205, USA \\ ${ }^{2}$ Laboratory of Pharmaceutical Sciences, Department of Biological Sciences and Biotechnology, Tsinghua University, \\ Beijing, 100084, China \\ ${ }^{3}$ McKusick-Nathans Institute of Genetic Medicine, Johns Hopkins University, Baltimore, MD 21205, USA
}

\begin{abstract}
Human lung cancer remains one of the deadliest diseases worldwide. New approaches are needed for improved lung cancer treatment. In this study, we found that $M$. tuberculosis culture supernatant (TB-SN) could inhibit human lung cancer cell proliferation through a caspase-dependent apoptosis pathway and induce cell cycle arrest in G1 phase. The active components responsible for the growth inhibitory activities were attributed to some proteins or protein complex with molecular weight more than $100 \mathrm{kD}$. These findings are significant and may provide new insight into a possible antagonism between $M$. tuberculosis and lung cancer and also have implications for development of an alternative approach for lung cancer treatment.
\end{abstract}

Keywords: M. tuberculosis, lung cancer, cell cycle, apoptosis.

\section{INTRODUCTION}

Cancer remains one of the most dangerous human diseases despite significant progress in cancer research and treatment in the past several decades. In 2007, it is estimated that 1.4 million people were diagnosed with cancer in the United States with 559,650 deaths [1]. According to the SEER Cancer Statistics Review, men have a $45 \%$ lifetime risk of being diagnosed with any type of cancer and women have a $38 \%$ chance of being diagnosed with any type of cancer [1]. Cancer rates could increase by as much as 50 percent to 15 million new cases by 2020 in the world [2]. The predicted increase will be mainly attributable to increases in the age of populations, the prevalence of smoking, and unhealthy diets [3]. In the year 2000, malignant tumors were implicated in nearly 12 percent of all human deaths worldwide from all causes. In many countries, more than 25 percent of all deaths are related to cancer [4]. In particular, human lung cancer is the second most common cancer and is the deadliest cancer that is the top cancer killer for both men and women. Although it accounts for just $13 \%$ of cancer cases, lung cancer is responsible for nearly one-third of all cancer deaths in men $(32 \%)$ and one-quarter of those in women $(25 \%)[4,5]$.

Currently, chemotherapy is the main standard treatment for human tumors. Although chemotherapy significantly improves symptoms and the quality of life of patients with

*Address correspondence to this author at the Department of Molecular Microbiology and Immunology, Bloomberg School of Public Health, Johns Hopkins University, 615 N. Wolfe Street, Baltimore, MD 21205, USA; Tel: 410-614-2975; Fax: 410-955-0105; E-mail: yzhang@jhsph.edu lung cancer, only modest increase in survival rate can be achieved [6-9]. One of the major reasons is the significant side effects and toxicity of current chemotherapy drugs. Several new alternative therapeutic approaches have been tried in the past few years. Bacteria can be used in combination with bacteriolytic therapy (COBALT), i.e., bacteria are administered together with conventional chemotherapeutic drugs to treat tumors [3]. These therapies take advantage of the ability of some bacteria to grow under hypoxic conditions. It is known that most advanced tumors have areas of poorly vascularized compartments creating hypoxic conditions, which render the cancer cells relatively resistant to conventional chemotherapy and radiation. Vogelstein and colleagues employed anaerobic bacteria Clostridium novyi in COBALT and achieved promising results for the treatment of cancer in a mouse model [10]. They created a strain of $C$. novyi devoid of its lethal toxin (C. novyi-NT) and intravenously injected $C$. novyi-NT spores into the avascular regions of tumors in mice. Most surrounding viable tumor cells were destroyed. When administered together with conventional chemotherapeutic drugs, they found that extensive hemorrhagic necrosis of tumors was developed and could result in significant and prolonged antitumor effects [10]. They also combined these bacteria with traditional radiotherapy and found it could also markedly improve the efficacy of radiotherapy [11]. Some bacteria can also be used as a delivery vector to effectively target tumors, which can replicate within tumors and inhibit tumor growth [12]. This new technology is called TAPET (Tumor Amplified Protein Expression therapy), an investigational anti-cancer gene, or protein, delivery vector [12]. Currently, Salmonella bacteria are often chosen for this purpose because they can proliferate rapidly, be easily modified genetically, and grow under both aerobic and 
anaerobic conditions. These bacterial strains have been designed to be highly selective for tumor tissue, relative to normal tissue, and to expand within the confines of the tumor to levels hundreds and even thousands of times greater than in normal tissue [13-14].

Some bacteria have been found to have significant antitumor activities. It has been reported that Lactobacillus species had significant cytotoxic effects on human bladder cancer in vitro and in vivo [15-17]. Attenuated Salmonella could also produce tumor growth inhibition [18]. Among all the bacteria that have anti-tumor activities, mycobacteria could be one of the most promising ones. The idea that mycobacteria could be used to treat human cancer has a long history. The first mycobacterium reported to have anti-tumor activity was BCG (derived from $M$. bovis, a member of the $M$. tuberculosis complex) [19], which is now widely used to treat human superficial bladder cancer $[20,21]$. It is thought that BCG might regulate human immune function and stimulates $\mathrm{T}$ lymphocytes to secrete cytokines (such as tumor necrosis factors, TNF) or other factors, which then kill tumor cells through a non-specific stander-by mechanism [21]. However, it is interesting to note that BCG is only effective in treating superficial surface located bladder cancer but not non-surface located bladder cancer, suggesting that BCG may also have direct effect on cancer cells besides its immunostimulatory role. Saitoh and Morales did provide evidence that BCG and BCG components could induce cancer cell apoptosis and exert cytotoxic effects [22]. It was also reported that other mycobacteria, such as $M$. phlei, or mycobacterial components, such as mycobacterial cell wall and DNA have certain anti-tumor activity [23, 24].

That mycobacteria could provide a promising way to treat human cancer, also receives support from occasional clinical observations. Some case reports about the relationship between $M$. tuberculosis and human cancer were published in the past few years [25, 26]. Interestingly, one report showed that a patient with lung cancer was completely recovered when he had tuberculosis [26]. It is likely that $M$. tuberculosis could exert a direct effect on cancer cells besides its stimulation of the immune system. M. tuberculosis is known to cause characteristic pathology of necrosis and liquefaction, which we may take advantage for lysing cancer cells. However, the real effects of $M$. tuberculosis on human lung cancer and its mechanism are still unclear and need further investigation. In this study, we found that TB culture supernatant (TB-SN) had growth inhibitory effect on various human cancer cells. In addition, we showed that TB-SN could significantly inhibit cancer cell proliferation and induce apoptosis and cell cycle arrest in lung cancer cell line A549. Finally, we demonstrated that the growth inhibitory activity of TB-SN was attributed to some proteins or protein complexes of high molecular weight.

\section{MATERIALS AND METHODS}

\section{Cell Cultures}

Human cancer cell lines A549, MCF7, SKBR3, AU565, HCC1937, Capan 1, BXPC3, ASPC1, HepG2 and
AGS were obtained from ATCC (American Type Culture Collection). Cells were grown in DMEM medium (GIBCO) supplemented with $10 \%$ fetal bovine serum, 100 units $/ \mathrm{ml}$ penicillin and $100 \mathrm{ug} / \mathrm{ml}$ streptomycin at $37^{\circ} \mathrm{C}$ in an incubator containing $5 \% \mathrm{CO}_{2}$. Avirulent $M$. tuberculosis strain $\mathrm{H} 37 \mathrm{Ra}$ was cultured in Sauton's medium. E. coli strain BL21 was cultured in M9 minimal medium.

\section{Preparation of $M$. tuberculosis Culture Supernatant}

M. tuberculosis strain H37Ra bacteria were inoculated into Sauton's medium and incubated at $37^{\circ} \mathrm{C}$ without shaking for one month. Supernatants of the mycobacterial culture were collected and concentrated by Series 8000 Stirred Cells with Ultrafiltration Membranes (5000 NMWL) (Millipore). Protein concentration was determined by using BCA protein assay kit (Pierce, USA). TB cellular fractions, including cell wall (CW), whole cell lysate (WCL), culture filtrate proteins (CFP), were obtained from Mycobacteria Research Laboratories, Colorado State University.

\section{Cell Growth Inhibition Assay}

MTT (3-(4,5-dimethyl-thiazol-2-yl)-2,5-diphenyl-tetrazolium bromide, Sigma) assay was used to evaluate the growth inhibitory activity of TB-SN on cancer cells. Briefly, cells were seeded at a concentration of $1.5 \times 10^{4}$ cells $/ \mathrm{ml}$ in a 96well plate. After overnight incubation, various concentrations of TB-SN as indicated were added and cells were incubated in a humidified atmosphere with $5 \% \mathrm{CO}_{2}$ for 3 days. Sauton's medium was used as a control and was found to have no growth inhibitory activity on cancer cells. Then, $20 \mu \mathrm{l}$ MTT solution $(4.14 \mathrm{mg} / \mathrm{ml})$ was added to each well and incubated at $37{ }^{\circ} \mathrm{C}$ for 4 hours. The medium was removed and the formazan was dissolved in DMSO and the optical density was measured at $590 \mathrm{~nm}$ using a Bio-assay reader (Bio-Rad, USA). The growth inhibition was calculated as: Growth inhibition = (control O.D.-sample O.D.)/control O.D.

\section{Cytotoxicity Assay}

The cytotoxic activity was assessed by detection of lactate dehydrogenase (LDH) release from dead cells after TB-SN treatment using Cytotoxicity Detection Kit (LDH) from Roche Applied Science following the manufacturer's protocol. Briefly, cell-free culture supernatants of TB-SN treated cancer cells were collected and transferred to microtiter plates. Then substrate mixture containing tetrazolium salts was added, and incubated for $3 \mathrm{~h}$ before the absorbance was measured at $490 \mathrm{~nm}$.

\section{Apoptosis Assay}

Vybrant $^{\circledR}$ Apoptosis Assay Kit \# 3 (FITC Annexin $\mathrm{V} /$ propidium iodide) was used to determine the percentage of cells undergoing apoptosis after TB-SN treatment as described by the manufacturer. Apoptotic cells were visualized by fluorescence microscopy and expressed as a percentage of apoptotic cells. A control without staining was also prepared.

\section{Microarray and Data Analysis}

Human lung cancer cell line A549 cells were collected and washed with PBS after treatment with TB-SN for 3 days and 
then lysed with Lysing Matrix D (Qbiogen) and Trizol LS. Subsequently, RNA was extracted according to the InVitrogen Trizol LS protocol with minor modification. The RNA was then purified following Qiagen RNeasy Total RNA Cleanup Protocol and quantified and qualified using Beckman spectrophotometer and RNA Nano LabChip analysis separately. For each sample, $7.5 \mu \mathrm{g}$ RNA was reversed transcribed to obtain the cDNA strands by Superscript II reverse transcriptase (Life Technologies) using T7-(dT) 24 primer containing a T7 RNA polymerase promoter. The cDNA generated was in vitro transcribed to generate complementary RNA (cRNA) using BioArray High Yield RNA Transcript Labeling Kit (ENZO) for microarray analysis.

GeneChip ${ }^{\circledR}$ Human Genome Focus Array (Affymetrix, Santa Clara, CA), which represent 8793 verified human sequences from the NCBI RefSeq database, were used in the microarray experiment performed according to the manufacturer's protocol. For each sample, ten microgram of cRNA was hybridized to the array according to the protocol. The eukaryotic hybridization controls were used to assess hybridization quality and array performance. The synthetic control oligo B2 provided alignment signals used by the software to position a grid over the array image. Ratios of signal for probe sets at $5 \mathrm{~V}$ and $3 \mathrm{~V}$ regions of housekeeping genes were calculated and monitored as an indication of transcript quality for each sample. After hybridization, the array was washed, stained and scanned at emission wavelength of 570nm using a confocal scanner (Affymetrix, Santa Clara, CA). The image data from the probe array scan was created and stored as data files for data analysis commenced with Absolute and Comparison analyses performed with Microarray suite 5.0 software. A fold change calculation was also computed to indicate the relative change of each transcript represented on the probe array. Further analyses with Data Mining Tool (Affymetrix) and GeneSpring (Silicon Genetics) were also performed.

\section{Cell Cycle Analysis}

Flow cytometry was used to quantitatively detect the cell-cycle distribution of cancer cells treated with TB-SN Cells were plated into $10-\mathrm{cm}$ tissue culture dishes 1 day before treatment with TB-SN. After treatment with TB-SN $(5 \mu \mathrm{g} / \mathrm{ml})$ for 3 days, cells were harvested, washed with PBS, fixed in $70 \%$ ethanol overnight at $20{ }^{\circ} \mathrm{C}$ for at least $12 \mathrm{~h}$. After two washes with phosphate buffered saline (PBS), the cells were incubated in RNase A/PBS (100 $\mu \mathrm{g} / \mathrm{ml})$ at $37{ }^{\circ} \mathrm{C}$ for $30 \mathrm{~min}$ and stained with $50 \mu \mathrm{g} / \mathrm{ml}$ propidium iodide (PI) by incubation at $4{ }^{\circ} \mathrm{C}$ for $15 \mathrm{~min}$. The stained cells (about $10^{5}$ cells) were analyzed by MoFlo flow cytometer (Dako Cytomation, Fort Collins, CO).

\section{Assessment of Caspase Activity}

ApoAlert ${ }^{\circledR}$ caspase fluorescent and colorimetric assays (BD Biosciences Clontech, CA, USA) were used to determine the activity of caspase- $3,-8$ and -9 . After being incubated with TB-SN for $48 \mathrm{~h}$, the cells were collected and lysed in ice-cold buffer. The homogenate was clarified by centrifugation at $12000 \mathrm{x} \mathrm{g}$ for $10 \mathrm{~min}$ at $4{ }^{\circ} \mathrm{C}$. Caspase activities in the supernatant were determined by cleavage of the specific chromophore-conjugated substrates. The substrate peptides of caspase-3, -8 and -9 (DEVD, IETD and LEHD) were conjugated to 7-amino-4-trifluoromethyl coumarin (AFC), p-nitroaniline (p-NA) and 7-amino-4-methyl coumarin (AMC), respectively. The release of AFC and AMC (for caspase-3 and -9) was measured by quantifying fluorescent intensity in a fluorescence spectrophotometer (Hitachi F-2500, Japan). Caspase-8 activity was determined by absorbance of pNA at $405 \mathrm{~nm}$ in a microplate reader

\section{Semi-Quantitative RT-PCR Analysis}

Semi-quantitative RT-PCR was carried out by using $50 \mathrm{ng}$ of RNA extracted from both treated and untreated A549 cancer cells. SuperScript III Platinum SYBR Green One-Step qRTPCR Kit (Invitrogen, Carlsbad, CA) was used. The confirmation experiments were preformed on an ABI Prism ${ }^{\circledR}$ 7000 machine (Applied Biosystems, Foster City, CA). The reverse transcription was carried out at $55^{\circ} \mathrm{C}$ for $20 \mathrm{~min}$, and the PCR reaction consisted of 40 cycles of denaturation ( $15 \mathrm{~s}$ at $95^{\circ} \mathrm{C}$ ), annealing $\left(30 \mathrm{~s}\right.$ at $\left.58{ }^{\circ} \mathrm{C}\right)$, and extension $(30 \mathrm{~s}$ at $72^{\circ} \mathrm{C}$ ). No-template reaction mixtures were included as negative controls. The primers used for STK6 were F (5'CCATGATGCTACCAGAGTCTAC CT3') and R (5'GACAGGGCATTTGCCAATTCTG 3'), and for OR10H3 they were F (5'CGTGGCTGTGC CCATCTTGT 3') and R (5'GGTGGATCACATTAGA CCCACAGA3').

\section{Statistical Analysis}

One-way analysis of variance (ANOVA) was performed to determine the significance between groups. A p-value of less than $0.05(\mathrm{p}<0.05)$ was considered as statistically significant.

\section{RESULTS}

\section{Specific Growth Inhibitory Effect of TB-SN on Human Lung Cancer Cell Line A549}

In order to determine the effects of $M$. tuberculosis on human cancer cells, the growth inhibitory effects of different components of $M$. tuberculosis on human lung cancer cell line A549 were tested, including cell wall $(\mathrm{CW}, 700 \mu \mathrm{g} / \mathrm{ml})$, whole cell lysate (WCL, $150 \mu \mathrm{g} / \mathrm{ml}$ ), culture filtrate proteins (CFP, $100 \mu \mathrm{g} / \mathrm{ml}$ ), live M. tuberculosis H37Ra (live TB, co-culture experiment), as well as $M$. tuberculosis culture supernatant (TB-SN, $5 \mu \mathrm{g} / \mathrm{ml}$ ). E. coli culture supernatant (E. coli-SN, 15 $\mu \mathrm{g} / \mathrm{ml}$ ) was used as a control. As shown in Fig. (1A), TB-SN had specific and high growth inhibitory effects on lung cancer A549 cells in a dose-dependent manner (Fig. 1B). The IC50 of TB-SN on A549 cells was $5 \mu \mathrm{g} / \mathrm{ml}$. In contrast, the E. coli culture supernatant did not have significant anticancer activity.

To validate the effect of the growth inhibition effects, TBSN was tested on 9 other cell lines including breast cancer cell lines MCF7, SKBR3, AU565, HCC1937, pancreatic cancer cell lines (Capan 1, BXPC3, ASPC1), liver (HepG2) and gastric (AGS) cancer cell lines. As shown in Fig. (2), TB-SN at a concentration of $5 \mu \mathrm{g} / \mathrm{ml}$ exhibited inhibitory effects on all the cancer cell lines tested, with growth inhibition rates ranging from $53 \%$ to $86 \%$. Taken together, these data suggest that TB-SN have significant growth inhibitory effect on a variety of different cancer cell lines. 

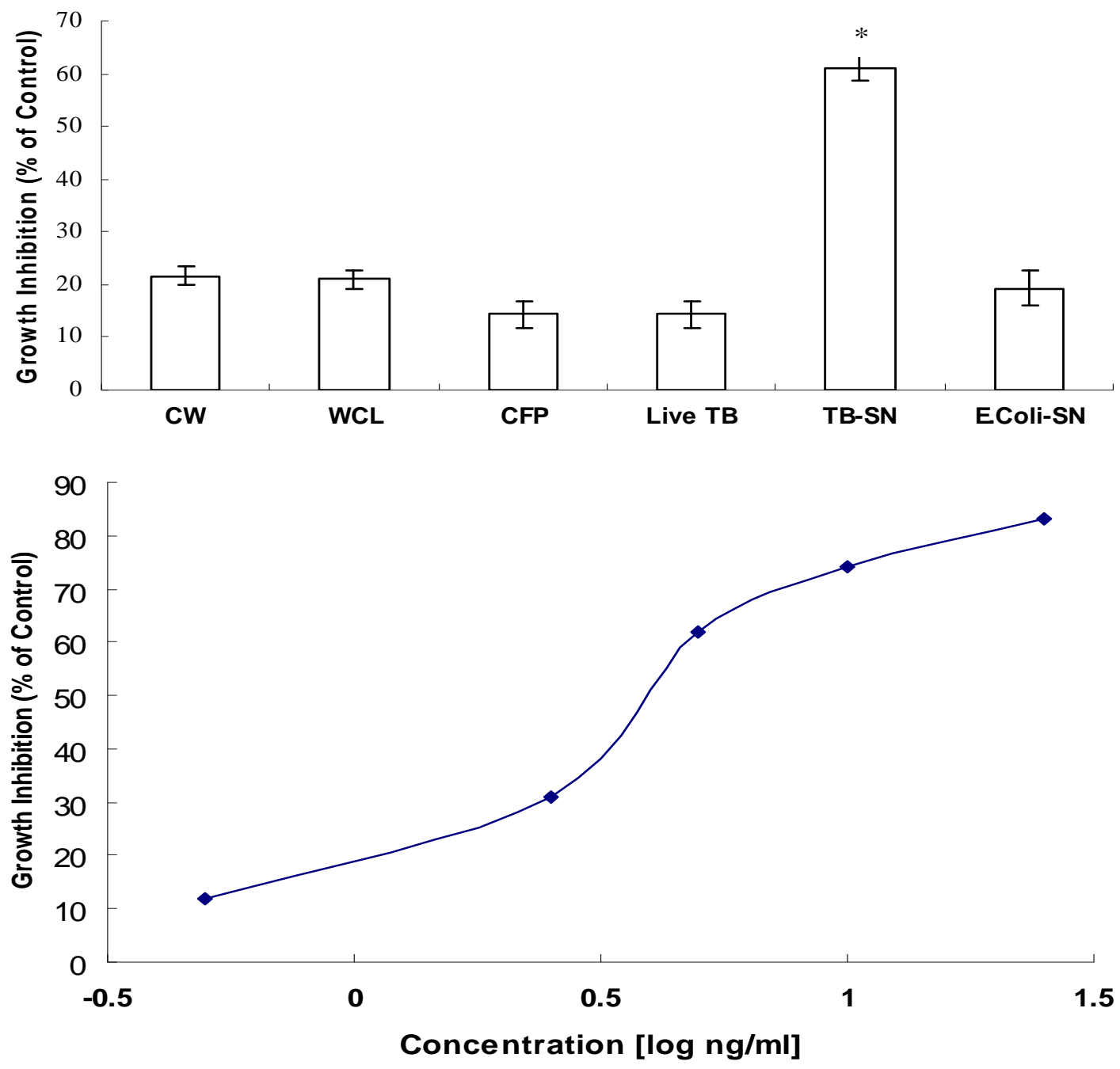

Fig. (1). Growth inhibitory effects of $M$. tuberculosis and E. coli components on A549 cancer cells. (A) different components of $M$. tuberculosis cultures, CW: cell wall, $700 \mu \mathrm{g} / \mathrm{ml}$; WCL: whole cell lysate, $150 \mu \mathrm{g} / \mathrm{ml}$; CFP: culture filtrate proteins, $100 \mu \mathrm{g} / \mathrm{ml}$; Live TB: live M. tuberculosis (co-culture experiment); TB-SN: culture supernatant, $5 \mu \mathrm{g} / \mathrm{ml}$; E. coli-SN: culture supernatant of E. coli, $15 \mu \mathrm{g} / \mathrm{ml}$; $($ B) different concentrations of TB-SN.

\section{Cytotoxic Effects of TB-SN on A549 Cells}

To determine the cytotoxic activity of TB-SN on A549 cells, the LDH cytotoxicity assay (Roche) was used. Untreated cells were used as negative control, and the cells treated with $1 \%$ Triton X-100 were used as positive control $(100 \%$ LDH release). Cytotoxicity was expressed as percentage of positive control. Indeed, TB-SN showed significant cytotoxic effect on A549 cells. Treatment of TB-SN at concentration of $5 \mu \mathrm{g} / \mathrm{ml}$ for 12 hours released $29.21 \%$ of total available $\mathrm{LDH}$.

\section{TB-SN Induced Apoptosis in a Caspase-Dependent Way}

A549 treated with TB-SN showed apoptosis phenotype, as indicated by appearance of small nuclear bodies (data not shown). To provide evidence that apoptosis is the main mechanism for the inhibition by TB-SN on A549 cells, we performed TUNEL apoptosis assay as described in Methods. Indeed, TN-SN significantly induced A549 cell apoptosis. As shown in Fig. (3), 36.8\% of TB-SN treated cells underwent apoptosis. In contrast, only $5.2 \%$ of cells underwent apoptosis in control group.

Apoptosis can be induced either by extrinsic pathway, which is mediated by Caspase 8, or by intrinsic pathway, which is mediated by Caspase 9. To further identify which apoptosis pathway was involved, Caspase 3/7, Caspase 8 and Caspase 9 were determined using Caspase-Glo ${ }^{\circledR}$ Assays kit from Promega. As shown in Fig. (4), caspase 3/7, 8 and 9 activities increased 6.61-, 4.72- and 1.98-fold separately after treatment with TB-SN for 48 hours at a concentration of 20 $\mu \mathrm{g} / \mathrm{ml}$. If A549 cells were pre-treated with DEVD-fmk, a 


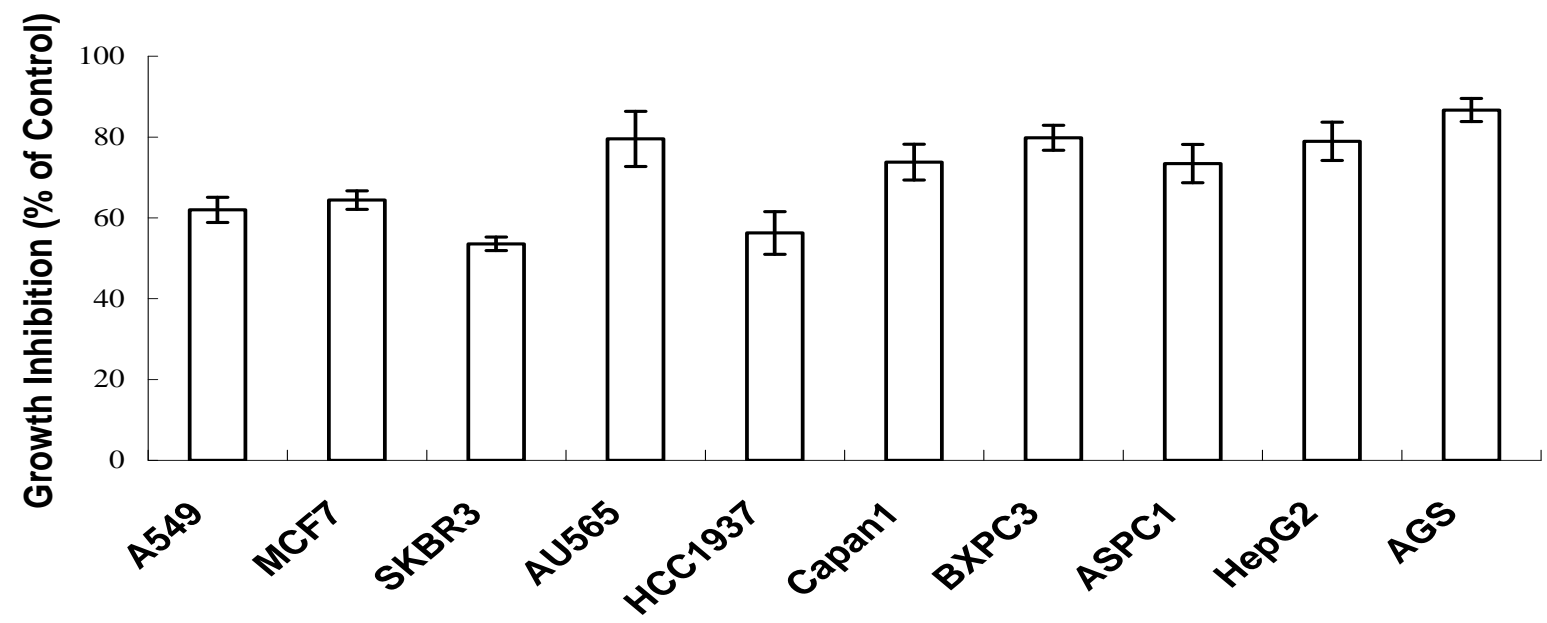

Fig. (2). Growth inhibitory effects of TB-SN ( $5 \mu \mathrm{g} / \mathrm{ml}$ for 3 days) on different human cancer cell lines.

PI

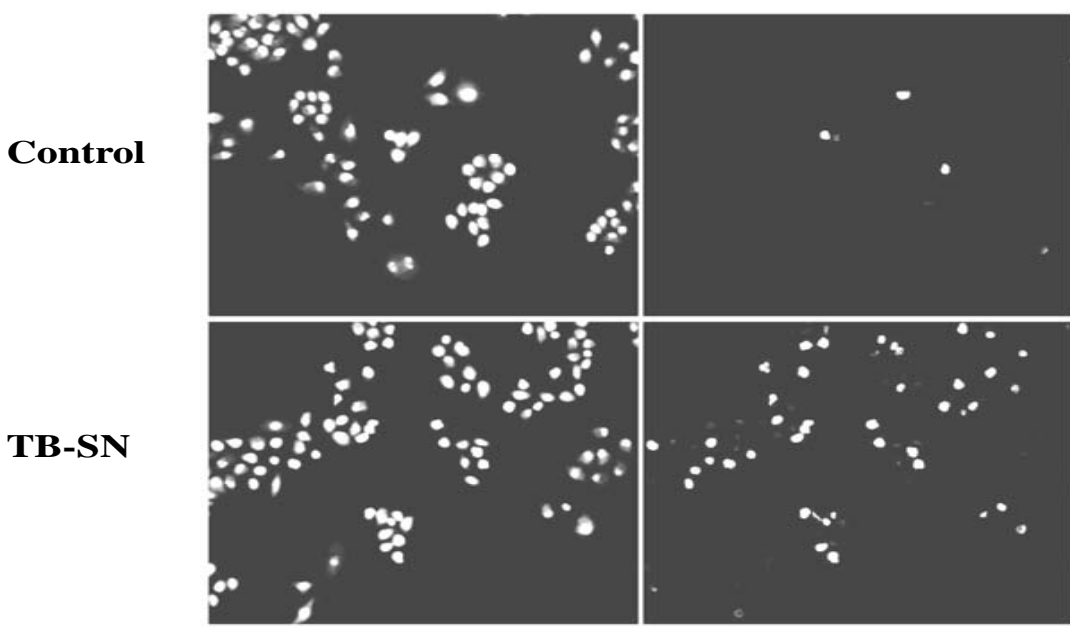

Merged

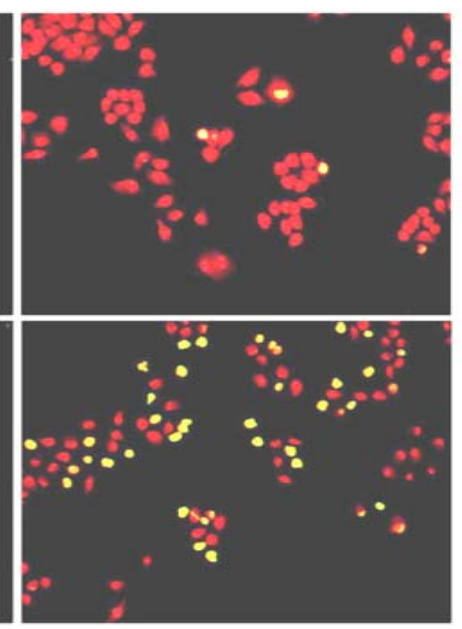

Fig. (3). TUNEL staining apoptosis assay of A549 cells treated with TB-SN for 48 hours at a concentration $5 \mu \mathrm{g} / \mathrm{ml}$.

known caspase 3 inhibitor, at $20 \mu \mathrm{M}$ for 12 hours and then treated with TB-SN for 48 hours, the percentage of apoptosis cells decreased from $36.8 \%$ (without pretreatment) to $13.4 \%$. These data suggested that TB-SN induces cancer cells apoptosis mainly in extrinsic, caspasedependent pathway.

\section{Microarray Analysis of TB-SN Induced Genes in Human Lung Cancer A549 Cells}

To assess the expression profiles in the TB-SN treated cancer cells and better understand the mechanism of TBSN induced cell apoptosis, Human Genome Focus Array (Affymetrix) chips were used in TB-SN treated A549 lung cancer cells for 4 hours and 5 days separately. Microarray results showed that TB-SN could dramatically change gene expression in A549 cells. After 4 hours TB-SN treatment, CCHCR1 was down-regulated nearly 200 fold, and RAB3A was up-regulated nearly 10 fold. After 5 days treatment with TB-SN, 7 genes were down-regulated and 8 genes were up-regulated over 10 fold as listed in Table 1. It is interesting to note that four cell cycle related genes including GSPT1 [27], CCND1 [28], STK6 [29] UBE2V1 [30], were dramatically down-regulated by more than 10 fold.

The first three of them are related to the G1/S transition of mitotic cell cycle. GSPT1 and STK6 are two G1/S transition regulation factors. Significant down-regulation of these two genes indicated that A549 cell cycle might be arrested at G1/S checkpoint. The down-regulation of CCND1 indirectly confirmed the assumption, as this gene expression is cell cycle regulated, low in G1/S and accumulated during G2/M. These data indicated that inducing cell cycle arrest could be another mechanism of the inhibitory effect of TB-SN on A549 cell proliferation. In addition, the gene expression profile also indicated that TB-SN treatment could affect cell signal transduction, especially the G-protein coupled receptor protein signaling pathway. At least four signal transduction related genes, including RAB3A, OR10H3, CALM1 [31], and NTS, were drastically up-regulated or down-regulated. The first three were G-protein coupled receptor protein signaling pathway factors. 
Table 1. Genes Regulated by TB-SN in A549 Cells by 5 Fold and Above in Microarray Analysis

\begin{tabular}{|c|c|c|}
\hline Genes & Definition & Folds Changed \\
\hline \multicolumn{3}{|c|}{4 Hours Treatment } \\
\hline CCHCR1 & Coiled-coil alpha-helical rod protein 1 & -192.65 \\
\hline RAB3A & RAB3A, member RAS oncogene family & 9.42 \\
\hline \multicolumn{3}{|c|}{5 Days Treatment } \\
\hline CCHCR1 & Coiled-coil alpha-helical rod protein 1 & 212.3 \\
\hline OR10H3 & Olfactory receptor, family 10 , subfamily $\mathrm{H}$, member 3 & 132.0 \\
\hline CLECSF2 & C-type lectin domain family 2 , member B & 15.33 \\
\hline FCGBP & Fc fragment of $\operatorname{IgG}$ binding protein & 15.30 \\
\hline NTS & Neurotensin & 12.70 \\
\hline $\mathrm{CP}$ & Ceruloplasmin (ferroxidase) & 12.30 \\
\hline FGG & Fibrinogen gamma chain & 11.80 \\
\hline SLC21A8 & Solute carrier family 21 (organic anion transporter), member 8 & 10.88 \\
\hline HLA-DMB & Major histocompatibility complex, class II, DM beta & 9.78 \\
\hline NUDT4 & Diphosphoinositol-polyphosphate diphosphatase activity & 9.47 \\
\hline PTGS2 & Prostaglandin-endoperoxide synthase activity & 8.97 \\
\hline FGB & Fibrinogen & 8.71 \\
\hline DF & Trypsin, complement factor D, hydrolase and chymotrypsin activity & 8.17 \\
\hline TFPI & Serine protease inhibitor activity & 8.13 \\
\hline $\mathrm{APOH}$ & Apolipoprotein $\mathrm{H}$ & 7.58 \\
\hline JDP1 & $\mathrm{J}$ domain containing protein 1 isoform $\mathrm{b}$, chaperone activity & 7.41 \\
\hline ANGPTL4 & Angiopoietin-like 4 protein isoform $b$ precursor & 6.68 \\
\hline TM4SF3 & Signal transducer activity & 6.63 \\
\hline ZNF277 & ZNF277 protein & 6.17 \\
\hline EI24 & Etoposide induced 2.4 isoform 2 & 5.99 \\
\hline LXN & Latexin, endogenous carboxypeptidase inhibitor & 5.98 \\
\hline FCGRT & IgG binding receptor activity & 5.74 \\
\hline $\mathrm{CPD}$ & Lysine (arginine) carboxypeptidase activity & 5.07 \\
\hline DNAJB9 & DnaJ chaperone & 5.01 \\
\hline STK6 & Serine/threonine-protein kinase 6; Aurora kinase A & -26.25 \\
\hline CCND1 & Cyclin D1 & -25.82 \\
\hline GSPT1 & Translation elongation factor activity, G1 to S phase transition 1 & -14.82 \\
\hline CALM1 & calmodulin 1 (phosphorylase kinase, delta) & -14.36 \\
\hline UBE2V1 & ubiquitin-conjugating enzyme E2 variant 1 & -12.10 \\
\hline UBE2V1 & Ubiquitin conjugating enzyme activity & -12.10 \\
\hline DNMT1 & DNA (cytosine-5-)-methyltransferase 1 & -11.20 \\
\hline DNMT1 & DNA (cytosine-5-)-methyltransferase activity & -11.20 \\
\hline WBP11 & WW domain binding protein 11 & -10.07 \\
\hline ZWINT & ZW10 interactor isoform b & -9.85 \\
\hline MCM6 & Minichromosome maintenance complex component 6 & -9.62 \\
\hline PTMS & Parathymosin & -9.55 \\
\hline
\end{tabular}


(Table 1) contd.....

\begin{tabular}{|c|c|c|}
\hline Genes & Definition & Folds Changed \\
\hline PAICS & Phosphoribosylaminoimidazole carboxylase & -8.64 \\
\hline HIST3H3 & DNA binding & -8.59 \\
\hline PHLDA1 & Apoptosis-associated nuclear protein PHLDA1 & -8.23 \\
\hline RFC5 & Replication factor C 5 isoform 2 & -7.90 \\
\hline PRC1 & Protein regulating cytokinesis 1 & -7.70 \\
\hline FEN1 & Flap structure-specific endonuclease 1 & -7.66 \\
\hline CHST6 & $\mathrm{N}$-acetylglucosamine 6-O-sulfotransferase activity & -7.48 \\
\hline ARCN1 & Protein transporter activity & -7.44 \\
\hline TYMS & Thymidylate synthetase & -7.13 \\
\hline NOLA1 & Nucleolar protein family A, member 1 & -7.11 \\
\hline NRG1 & Neuregulin 1 type IV fetal C beta $1 \mathrm{a}$ & -7.09 \\
\hline PPIF & Peptidylprolyl isomerase F (cyclophilin F) & -7.09 \\
\hline HNRPD & Heterogeneous nuclear ribonucleoprotein D & -6.83 \\
\hline RAD23B & UV excision repair protein RAD23 homolog B & -6.78 \\
\hline YWHAB & Tyrosine 3-monooxygenase activation protein & -6.72 \\
\hline HNRPM & Heterogeneous nuclear ribonucleoprotein $\mathrm{M}$ isoform $\mathrm{b}$ & -6.62 \\
\hline SMC1L1 & Structural maintenance of chromosomes $1 \mathrm{~A}$ & -6.55 \\
\hline $\mathrm{DKC1}$ & Dyskerin, H/ACA ribonucleoprotein complex subunit 4 & -6.45 \\
\hline TARDBP & TAR DNA binding protein & -6.33 \\
\hline CSE1L & Chromosome segregation 1-like protein & -6.07 \\
\hline UNG & Uracil DNA N-glycosylase activity & -6.06 \\
\hline PFDN1 & Chaperone activity, transcription factor activity & -6.03 \\
\hline MCM5 & Minichromosome maintenance complex component 5 , & -5.71 \\
\hline TACC3 & Transforming acidic coiled-coil containing protein 3 & -5.70 \\
\hline FTSJ2 & Putative ribosomal RNA methyltransferase 2 & -5.70 \\
\hline CDC6 & Cell division cycle 6 protein & -5.69 \\
\hline HNRPU & Heterogeneous nuclear ribonucleoprotein $\mathrm{U}$, isoform a & -5.64 \\
\hline TPM4 & Actin binding, structural constituent of muscle & -5.63 \\
\hline SQSTM1 & Sequestosome 1, ubiquitin-binding protein $\mathrm{p} 62$ & -5.53 \\
\hline KIF20A & kinesin family member $20 \mathrm{~A}$ & -5.32 \\
\hline MCCC2 & Methylcrotonoyl-Coenzyme A carboxylase 2 (beta) & -5.30 \\
\hline FER1L3 & Myoferlin isoform b & -5.18 \\
\hline RRM1 & Ribonucleoside-diphosphate reductase M1 chain & -5.15 \\
\hline USP39 & ubiquitin specific protease 39 & -5.11 \\
\hline $\mathrm{CDC} 20$ & Cell division cycle 20 & -5.00 \\
\hline
\end{tabular}




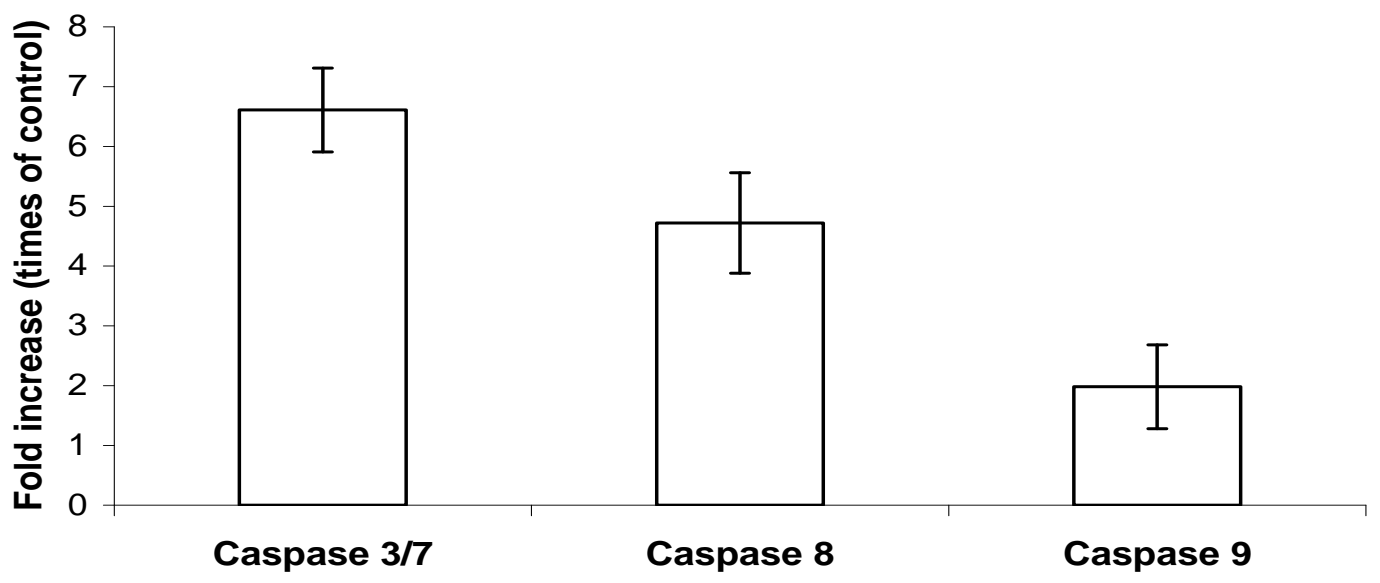

Fig. (4). Changes in caspase 3/7, 8 and 9 activities in A549 cancer cells after treatment with $20 \mu \mathrm{g} / \mathrm{ml}$ TB-SN for 48 hours.

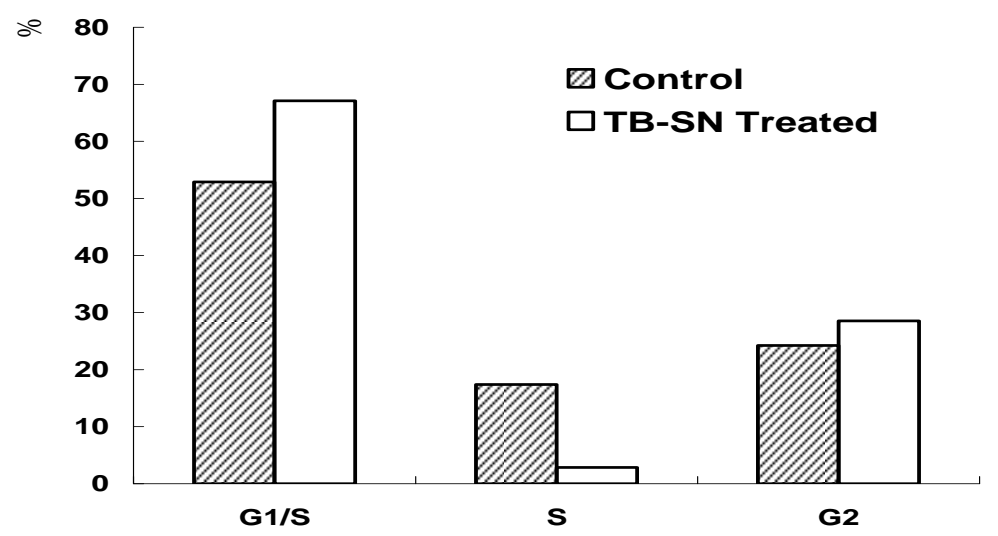

Fig. (5). Flow cytometry cell cycle analysis after TB-SN treatment ( $5 \mu \mathrm{g} / \mathrm{ml}$ for 3 days).

To confirm the gene expression changes after TB-SN treatment demonstrated in microarray assay, STK6 and OR10H3 expression levels were detected using quantitative real-time PCR. As summarized in Table 2, the qRTPCR data was consistent with cDNA array data.

Table 2. Relative Expression Changes of AURKA/STK6 and OR10H3 after TB-SN Treatment for 4 and 120 Hours in Semi-Quantitative RT-PCR Analysis

\begin{tabular}{|c|c|c|}
\hline Time /h & AURKA/STK6 & OR10H3 \\
\hline \hline 4 & $-1.76 \pm 0.34$ & $+1.37 \pm 0.17$ \\
\hline 120 & $-6.58 \pm 0.57$ & $+9.07 \pm 0.06$ \\
\hline
\end{tabular}

\section{Cell Cycle Analysis of TB-SN Treated Cancer Cells}

To confirm that induction of cell cycle arrest is one of the mechanisms for inhibitory effect of TB-SN on A549 cell proliferation, flow cytometry cell cycle analysis was performed. A549 cells were first treated with TB-SN and then cell cycle was analyzed by using MoFlo Flow Cytometer. As expected, TB-SN induced cells arrest at G1/S checkpoints, with G1/S phase cell population increasing from $52.90 \%$ to $67.12 \%$ (Fig. 5). It seems that the increase of G1/S phase population came mainly from $\mathrm{S}$ phase since the $\mathrm{S}$ phase decreased from $17.38 \%$ to $2.28 \%$ after treatment and no significant change was observed for G2 phase.

\section{Identification of Antitumor Activities in TB-SN}

We attempted to identify the active components in the TBSN responsible for the growth inhibitory effect on A549 cells. The culture supernatant could contain macromolecules like proteins and polysaccharides as well as small organic molecules including amino acids, monosaccharide and nucleic acids, and inorganic molecules. Among them, proteins are heat-sensitive, while other components are heat-resistant. To determine if the active component of TB-SN is protein, we compared the growth inhibition effect of TB-SN with and without heat treatment at $100{ }^{\circ} \mathrm{C}$ for 10 minutes. Interestingly, TB-SN lost most of the activity after heating (Fig. 6). Similar results were obtained when proteins were removed from TBSN by TCA precipitation (Fig. 6). These data indicated that proteins are the main component in $\mathrm{TN}-\mathrm{SN}$, which causes the growth inhibitory effect on A549 cells. To identify the molecule weight of the protein or protein complex responsible for the inhibitory effect of TB-SN, Amicon Ultra Centrifugal 


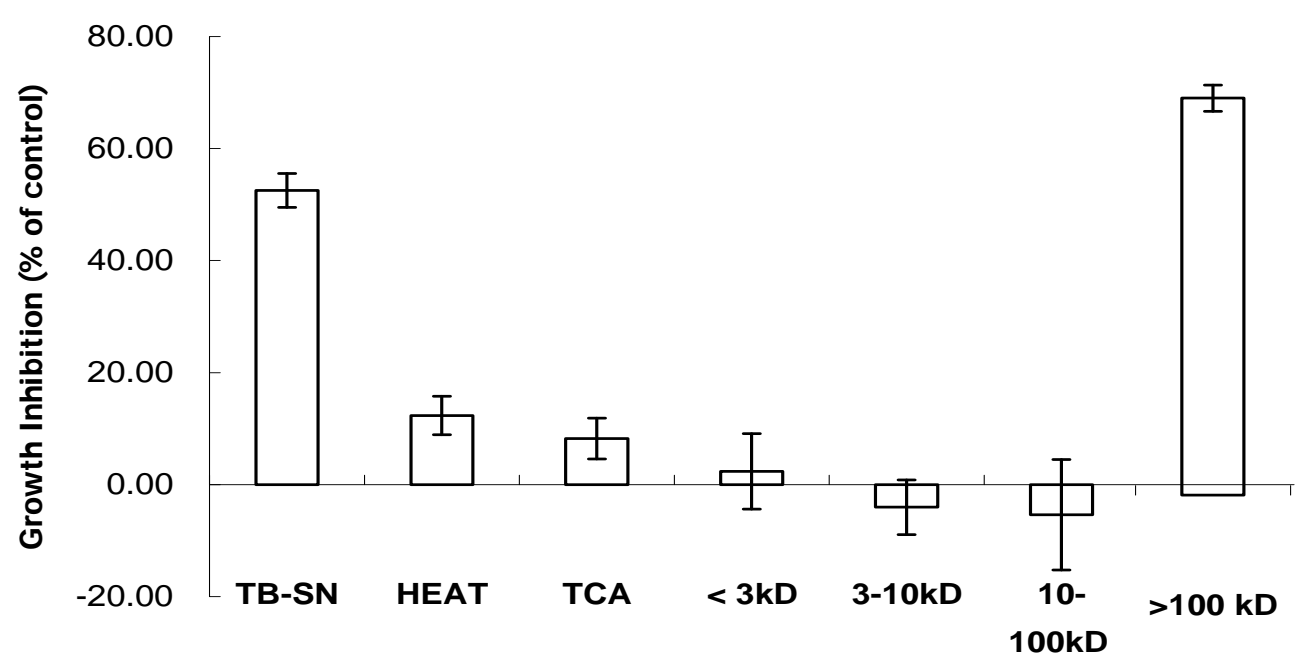

Fig. (6). Growth inhibitory effects of various fractions of TB-SN on A549 cells. (1) TB-SN: $5 \mu \mathrm{g} / \mathrm{ml}$; (2) HEAT: TB-SN treated at $100{ }^{\circ} \mathrm{C}$ for 10 minutes; (3) TCA: TB-SN treated with TCA; (4) $<3 \mathrm{kD}$ : Fractions of with MW less than $3 \mathrm{kD}$; (5) 3-10 kD: Fractions with MW between 3 and $10 \mathrm{kD}$; (6) 10-100 kD: Fractions of TB-SN with MW between 10 and 100 kD; (7) >100 kD: Fractions of TB-SN with MW more than $100 \mathrm{kD}$. Untreated cells were used as control.

Separation System (Millipore) was used to fractionate TBSN into several different molecular weights, including less than $3 \mathrm{kD}, 3 \sim 10 \mathrm{kD}, 10 \sim 100 \mathrm{kD}$ and more than $100 \mathrm{kD}$. These fractions were further tested on A549 cells for growth inhibitory effect. As shown in Fig. (6), the fractions with molecular weight more than $100 \mathrm{kD}$ contained most of the activities of TB-SN. Taken together, these data indicated that the active component of TB-SN could be proteins or protein complex with molecular weight more than $100 \mathrm{kD}$.

\section{DISCUSSION}

It has remained controversial concerning the possible association between $M$. tuberculosis and lung cancer since the middle of 19th century. Some authors claim that tuberculosis promotes development of cancer [32], while others assert that tuberculosis and cancer are antagonistic [33]. Therefore, it is difficult to assess the relationship of $M$. tuberculosis and lung cancer simply based on their coexistence. In this study, we demonstrated that TB-SN could cause significant growth inhibition in various human cancer cell lines including human lung cancer cell line A549 at low concentrations. The IC50 of TB-SN in A549 lung cancer cell is about $5 \mu \mathrm{g} / \mathrm{ml}$. LDH release assay revealed that TB-SN had cytotoxic effects on A549 lung cancer cells. At a concentration of $5 \mu \mathrm{g} / \mathrm{ml}$, TB-SN had about $29.2 \%$ of cytotoxicity of Triton X-100 (1\%), a positive control that can cause almost all cell death through cytotoxic effect. In addition, we found that TB-SN could induce cell apoptosis. At a concentration of $5 \mu \mathrm{g} / \mathrm{ml}$, TBSN could induce about $36.8 \%$ A549 cancer cells to undergo apoptosis as shown in the TUNEL apoptosis assay. Further studies revealed that this apoptosis was induced through an extrinsic caspase-dependent pathway, as TB-SN at $20 \mu \mathrm{g} / \mathrm{ml}$ could increase caspase $3 / 7$ and 8 levels by 6.61 - and 4.72 -fold, respectively, after treatment with TB-SN for 5 hours. Caspase 3 is a critical effector caspase in caspase-dependent apoptosis pathway [34]. When A549 cancer cells were pretreated with caspase 3 inhibitor DEVD-fmk, the apoptosis percentage decreased significantly. Since reduction in cell growth and induction in cell death are two major means to inhibit tumor growth [35], TB-SN has good potential to be developed into useful treatment for human cancers.

cDNA microarray is a powerful tool to identify mechanism of action of drugs [36]. In this study, cDNA microarray demonstrated that TB-SN treatment dramatically changed the profile of gene expression in A549 lung cancer cells. Altogether, 196 functionally related genes were altered after 4 hour treatment and 371 genes after 5 day treatment. Among these, 2 genes were found to change more than 5 -fold after 4 hour treatment, and 15 genes more than 10 -fold after 5 day treatment with 7 genes down-regulated and 8 genes upregulated. The gene expression pattern of A549 cancer cells after TB-SN treatment indicated that TB-SN might work through several mechanisms. First, TB-SN may activate Gprotein coupled receptor signaling pathway, since OR10H3 was dramatically up-regulated over 132-fold after 5 day treatment. Also, NTS and CALM1 involved in signal transduction were induced over 10-fold. Second, TB-SN might regulate cell cycle since four genes including GSPT1, CCND1, STK6, UBE2V1 were down-regulated significantly over 10fold. These data are consistent with the cell cycle analysis by flow cytometry, which demonstrated that TB-SN could induce A549 cancer cells arrest at G1/S checkpoint. Third, TB-SN may induce apoptosis, as expression of 13 apoptosis related genes was altered after TB-SN treatment. This result is consistent with the TUNEL staining result. All these results indicated that TB-SN could activate G-protein coupled signaling pathway and induce cell apoptosis and cell cycle arrest at G1/S checkpoint as potential mechanisms of action.

Chemical and physical analyses suggest that the active components in TB-SN responsible for inhibition of cell proliferation were protein or protein complexes with molecular weight greater than $100 \mathrm{kD}$. Further work is needed to identify the active components. Also, the gene expression profile of CCHCR1 gene in TB-SN treatment was remarkable, since it 
was down-regulated about 193 -fold after 4 hours treatment and up-regulated over 200-fold after 5 day treatment. However its function is still unclear and further studies are needed to clarify its role in TB-SN induced anticancer activity

\section{ACKNOWLEDGEMENTS}

We thank Anne Jedlicka and Alan Scott for assistance with the microarray experiment and Hao Zhang for the flow cytometry analysis. We acknowledge the receipt of M. tuberculosis components from Mycobacteria Research Laboratories, Colorado State University. Chao Ma was supported by China Scholarship Council. YZ was supported by AI44063 and in part by Ho Ching Yang Memorial Faculty Fellowship in Cancer Prevention from Johns Hopkins Bloomberg School of Public Health.

\section{REFERENCES}

[1] SEER Cancer Statistics Review, 1975-2005. Available at: http://seer.cancer.gov/csr/1975_2005/

[2] Bernard W, Stewart PK. World cancer report. Lyon: IARC 2003.

[3] Dang LH. Combination bacteriolytic therapy for the treatment of experimental tumors. Proc Natl Acad Sci USA 2001; 98: 1515560.

[4] Global cancer rates could increase by $50 \%$ to 15 million by 2020 Available at: http://www.who.int/mediacentre/releases/2003/pr27/en/

[5] Cancer Deaths to Decline in 2004. Available at: http://www. cancer.org/docroot/NWS/content/NWS_1_1x_Cancer_Deaths_to Decline_in_2004.asp.

[6] ten Bokkel Huinink WW. Single-agent gemcitabine: an active and better tolerated alternative to standard cisplatin-based chemotherapy in locally advanced or metastatic non-small cell lung cancer. Lung Cancer 1999; 26: 85-94.

[7] Sandler AB. Phase III trial of gemcitabine plus cisplatin versus cisplatin alone in patients with locally advanced or metastatic non-small-cell lung cancer. J Clin Oncol 2000; 18: 122-30.

[8] Bonomi P. Comparison of survival and quality of life in advanced non-small-cell lung cancer patients treated with two dose levels of paclitaxel combined with cisplatin $v s$. etoposide with cisplatin: results of an Eastern Cooperative Oncology Group trial. J Clin Oncol 2000; 18: 623-31

[9] Chou JY. Investigation of anticancer mechanism of thiadiazolebased compound in human non-small cell lung cancer A549 cells. Biochem Pharmacol 2003; 66: 115-24.

[10] Saitoh H. BCG effects on telomerase activity in bladder cancer cell lines. Int J Clin Oncol 2002; 7: 165-70.

[11] Bettegowda C. Overcoming the hypoxic barrier to radiation therapy with anaerobic bacteria. Proc Natl Acad Sci USA 2003; 100: 15083-88.

[12] Zheng LM. Tumor amplified protein expression therapy: Salmonella as a tumor-selective protein delivery vector. oncology research featuring preclinical and clinical cancer therapeutics 2001; 12: 127-35.

[13] Bermudes D. Tumor-targeted Salmonella. Highly selective delivery vectors. Adv Exp Med Biol 2000; 465: 57-63.
[14] Bermudes D. Live bacteria as anticancer agents and tumor-selective protein delivery vectors. Curr Opin Drug Discov Devel 2002; 5: 194-

[15] Seow SW, Mohamed AAK, Lee YK, Bay BH. Lactobacillus species is more cytotoxic to human bladder cancer cells than mycobacterium bovis (Bacillus Calmette-Guerin). J Urol 2002; 168: 2239-2239.

[16] Asano M. Antitumor activity of Lactobacillus casei (LC 9018) against experimental mouse bladder tumor (MBT-2). J Urol 1986; 136: 719-21.

[17] Takahashi T. Antitumor effects of the intravesical instillation of heat killed cells of the Lactobacillus casei strain Shirota on the murine orthotopic bladder tumor MBT-2. J Urol 2001; 166: 2506-11.

[18] Luo X. Antitumor effect of VNP20009, an attenuated Salmonella, in murine tumor models. Oncol Res 2000; 12: 501-08.

[19] Morales A. Intracavitary Bacillus Calmette-Guerin in the treatment of superficial bladder tumors. J Urol 1976; 116: 180-3.

[20] O'Donnell MA. Combined bacillus Calmette-Guerin and interferon use in superficial bladder cancer. Expert Rev Anticancer Ther 2003; 3: 809-21.

[21] Witjes JA. Mycotic aneurysm of the popliteal artery as a complication of intravesical BCG therapy for superficial bladder cancer. Case report and literature review. Urol Int 2003; 71: 430-2.

[22] Yoo YC. Inhibitory effect of BCG cell-wall skeletons (BCG-CWS) emulsified in squalane on tumor growth and metastasis in mice. Arch Pharm Res 2002; 25: 522-7.

[23] Filion MC. Mycobacterium phlei cell wall complex directly induces apoptosis in human bladder cancer cells. Br J Cancer 1999; 79: 22935 .

[24] Kabbaj M. Anticancer activity of mycobacterial DNA: effect of formulation as chitosan nanoparticles. J Drug Target 2001; 9: 317-28.

[25] Chinesta AJ. Mycobacterium kansasii lung infection and synchronous bronchopulmonary carcinoma. An Med Intern 2002; 19: 186-8.

[26] Mariani F. Tuberculosis and lung cancer. An interesting case study. Arch Chest Dis 2001; 56: 30-32.

[27] Lee JA. G1 to S phase transition protein 1 induces apoptosis signalregulating kinase 1 activation by dissociating 14-3-3 from ASK1. Oncogene 2008; 27: 1297-305

[28] Aggarwal P. Nuclear accumulation of cyclin D1 during S phase inhibits Cul4-dependent Cdt1 proteolysis and triggers p53-dependent DNA rereplication. Genes Dev 2007; 21: 2908-22.

[29] Hongyi Z. Tumour amplified kinase STK15/BTAK induces centrosome amplification, aneuploidy and transformation. Nat Genetics 1998; 20: 189-93.

[30] Syed NA, Uev1A, a ubiquitin conjugating enzyme variant, inhibits stress-induced apoptosis through NF-kappaB activation. Apoptosis 2006; 11: 2147-57

[31] Colomer J. Expression of calmodulin and calmodulin binding proteins in lymphoblastoid cells. J Cell Physiol 1994; 159: 542-50.

[32] Dacosta NA. Association of lung carcinoma and tuberculosis. J Postgrad Med 1991; 37: 185-89.

[33] Cicenas S. Lung cancer in patients with tuberculosis. World J Surg Oncol 2007; 5:22.

[34] Yacobi G. Gonadotropins enhance caspase-3 and -7 activity and apoptosis in the theca-interstitial cells of rat preovulatory follicles in culture. Endocrinology 2004; 145:1943-51.

[35] Huang KC. Phyllanthus urinaria triggers the apoptosis and Bcl-2 down-regulation in Lewis lung carcinoma cells. Life Sci 2003; 72 1705-16.

[36] Huang KC. Superoxide dismutase as a target for the selective killing of cancer cells. Nature 2000; 407: 390-5.

(C) Ma et al.; Licensee Bentham Open.

This is an open access article licensed under the terms of the Creative Commons Attribution Non-Commercial License (http://creativecommons.org/licenses/by$\mathrm{nc} / 3.0 /$ ), which permits unrestricted, non-commercial use, distribution and reproduction in any medium, provided the work is properly cited 